\title{
Fetal exposure to a maternal low-protein diet during mid-gestation results in muscle-specific effects on fibre type composition in young rats
}

\author{
Joanne E. Mallinson, Dean V. Sculley, Jim Craigon, Richard Plant, Simon C. Langley-Evans \\ and John M. Brameld* \\ Division of Nutritional Sciences, School of Biosciences, University of Nottingham, Sutton Bonington, Loughborough \\ LE12 5RD, UK
}

(Received 11 August 2006 - Revised 20 December 2006 - Accepted 29 January 2007)

\begin{abstract}
This study assessed the impact of reduced dietary protein during specific periods of fetal life upon muscle fibre development in young rats. Pregnant rats were fed a control or low-protein (LP) diet at early (days 0-7 gestation, LPEarly), mid (days 8-14, LPMid), late (days 15-22, LPLate) or throughout gestation (days 0-22, LPAll). The muscle fibre number and composition in soleus and gastrocnemius muscles of the offspring were studied at 4 weeks of age. In the soleus muscle, both the total number and density of fast fibres were reduced in LPMid females $(P=0.004$ for both, Diet $\times$ Sex $\times$ Fibre type interactions), while both the total number and density of glycolytic (non-oxidative) fibres were reduced in LPEarly, LPMid and LPLate (but not LPAll) offspring compared with controls $(P<0.001$ for both, Diet $\times$ Fibre type interaction). In the gastrocnemius muscle, only the density of oxidative fibres was reduced in LPMid compared with control offspring $(P=0 \cdot 019$, Diet $\times$ Fibre type interaction), with the density of slow fibres being increased in LPAll males compared with control $(P=0 \cdot 024$, Diet $\times \operatorname{Sex} \times$ Fibre type interaction). There were little or no effects of maternal diet on fibre type diameters in the two muscles. In conclusion, a maternal low-protein diet mainly during mid-pregnancy reduced muscle fibre number and density in 4-week-old rats, but there were muscle-specific differences in the fibre types affected.
\end{abstract}

Skeletal muscle: Prenatal undernutrition: Rat: Fetal programming: Protein

During muscle development, the commitment of precursor cells to form myoblasts in early embryonic life is followed by differentiation into myotubes and maturation into muscle fibres (for reviews see Brameld et al. 1998, 2003; Maltin et al. 2001). The first fibres formed are defined as primary fibres, with secondary fibres forming slightly later, around the primary fibres. In general, the primary fibres tend to become type I (slow, oxidative) fibres, while secondary fibres tend to become type II (IIA: fast, mixed oxidative and glycolytic; IIB: fast, glycolytic) fibres. In rats, the development of primary fibres takes place around $14-16 \mathrm{~d}$ of fetal life and secondary fibres around 17$19 \mathrm{~d}$ of fetal life (Wilson et al. 1988), although differences between muscles probably exist.

A number of studies (Nordby et al. 1987; Wilson et al. 1988; Dwyer et al. 1994, 1995; Bayol et al. 2004; Fahey et al. 2005) have examined the effect of prenatal nutrition on muscle fibre development and composition in a variety of mammalian species. In general, prenatal undernutrition increases the proportion of type I (slow) fibres and reduces the proportion of type II (fast) fibres in young offspring, due to a reduction in the numbers of secondary (fast) fibres formed. For example, Wilson et al. (1988) showed a reduction in secondary fibres in both soleus and lumbrical muscles of the newborn rat when the maternal diet was restricted to $30 \%$ of the control animals. Suckling rats, whose mothers were re-fed to the level of controls after birth and during lactation, showed no subsequent change in the numbers of fibres in soleus, but the fibre number in the lumbrical muscles was increased following refeeding at $28 \mathrm{~d}$ of age (Wilson et al. 1988). This suggests that the numbers of fibres within the rat soleus muscle are fixed at birth and any changes induced in the total number of fibres during muscle development persist in the older animal.

Few studies have examined the effect of maternal undernutrition, or specific components of the diet (e.g. protein content), at different stages of gestation on muscle fibre composition in the resulting young. We recently demonstrated (Fahey et al. 2005) that restriction of the whole diet targeted specifically to the proliferation period (days 30-70 of gestation) prior to major (secondary) fibre formation in fetal sheep results in reduced numbers of secondary (fast) fibres in neonatal lambs ( 2 weeks of age). We therefore postulated that a dietary challenge prior to secondary fibre formation in the rat (i.e. days $8-21$, which equates to mid- to late gestation) would result in reduced numbers of secondary (fast) fibres formed and thereby change the fibre composition of muscles in young rats. We also set out to investigate whether a dietary challenge prior to primary fibre formation (i.e. days 7-14 or mid-gestation in the rat) might also result in reduced numbers of slow fibres.

\footnotetext{
Abbreviations: LP, low protein.

* Corresponding author: Dr John M. Brameld, fax +44 (0)115 951 6122, email John.Brameld@nottingham.ac.uk
} 
The aim of the present study was therefore to examine the impact of a prenatal low-protein (LP) diet at different stages of pregnancy on the fibre composition of rats at weaning. The impact of the dietary challenge was assessed in soleus, a predominantly slow muscle, and gastrocnemius, a predominantly fast muscle. We hypothesised that prenatal LP exposure would decrease the number of secondary (fast) fibres within soleus and gastrocnemius muscles and that mid- to late pregnancy would be the critical time of exposure.

\section{Materials and methods}

\section{Chemicals and reagents}

Unless indicated otherwise in the text all chemicals were of reagent grade and were purchased from Sigma-Aldrich (Poole, UK).

\section{Animals}

The experiments in the present study were performed in accordance with the Animals (Scientific Procedures) Act 1986 and were licensed by the Home Office. Animals were held under temperature-controlled conditions on a $12 \mathrm{~h}$ light-dark cycle. The animals had ad libitum access to food and water at all times. Fifty-seven virgin female Wistar rats (Harlan Ltd, Belton, UK) were mated at weights between 250 and $300 \mathrm{~g}$. Upon confirmation of mating by the appearance of a semen plug on the cage floor, the rats were allocated to be fed either a control diet (18\% casein), or a LP diet (9\% casein), as described previously (Langley-Evans et al. 1996). The full composition of the diets is published elsewhere (Langley-Evans et al. 1996). The diets were isoenergetic, the difference in energy between the control and LP diets being made up with additional carbohydrate in a ratio of 2:1 starch-sucrose (w/w). LP feeding was targeted at single weeks in gestation days 0-7 (LPEarly), days 814 (LPMid) and days 15-22 (LPLate) and also fed throughout gestation (days 0-22, LPAll). Eight dams (one control, one LPAll, three LPEarly and three LPMid) failed to deliver at the end of gestation. As described previously (Bellinger et al. 2006), maternal protein restriction had no effect on litter size. The early period (days $0-7$ ) corresponds to the embryonic phase of development in the rat and in fact embryos only implant at around day 4.5 (Kwong et al. 2000). The mid-gestation period (days 8-14) largely corresponds to the period of organogenesis, whilst late gestation (days 15-22) is the period of most rapid growth and differentiation of key structures. By feeding at these targeted periods it is possible to identify when nutritional programming occurs and this can provide important indicators of potential mechanisms.

At delivery of litters, all mothers were transferred to standard laboratory chow diet (B\&K Universal rat and mouse diet, $20 \%$ protein, $3 \%$ fat) and the litters were culled to a maximum of eight pups to minimise variation in the nutrition of the pups during suckling. None of the dams gave birth to fewer than ten pups. The protein restriction applied in the present study is mild. Hepatic glutathione concentration is one of the most sensitive indices of protein status in the rat and we have recently shown that this is unaffected in rats fed the
$9 \%$ casein diet throughout pregnancy (Langley-Evans et al. 2006). Recovery from such a mild protein restriction will be very rapid, and indices such as glutathione have been shown to normalise within $24 \mathrm{~h}$ of even severe food restriction (Colomb et al. 1995). Although not measured in the present study, we have previously shown that the protein content of milk from rats fed LP throughout pregnancy was similar to that of controls at $12 \mathrm{~h}$ post-partum (Bellinger \& LangleyEvans, 2003). Hence, the offspring of the control and LPfed dams differed only in terms of their prenatal nutritional exposures. At 4 weeks of age, one male and one female from each litter were killed, using a rising concentration of carbon dioxide, prior to dissection of muscles. Data relating to other aspects of the present study have been published elsewhere (Bellinger et al. 2006; Langley-Evans \& Sculley, 2006).

\section{Histochemistry}

Soleus and gastrocnemius muscles from the right hindlimb were dissected from the 4-week-old rats. Muscles were frozen in isopentane cooled with liquid nitrogen and stored at $-80^{\circ} \mathrm{C}$ until analysis. Serial $10 \mu \mathrm{m}$ thick sections were cut from the mid-belly region of the muscle on a cryostat at $-20^{\circ} \mathrm{C}$. Sections were placed on Superfrost ${ }^{\circledR}$ Plus slides and left to defrost to allow the sections to adhere to the slides. Sections were stored at $-80^{\circ} \mathrm{C}$ until histochemical analysis was performed.

\section{Alkaline myofibrillar ATPase staining}

Alkaline myofibrillar ATPase staining was carried out according to Wegner et al. (2000). This stain allows for the discrimination between slow and fast fibre types, with fast (type II) fibre types staining blue and slow (type I) fibres failing to stain (i.e. are white). Briefly, the sections were left at room temperature for $2 \mathrm{~min}$ before being washed twice for $1 \mathrm{~min}$ each in Tris- $\mathrm{Ca}^{2+}$ pre-rinse solution and incubated for $5 \mathrm{~min}$ in alkaline pre-incubation solution containing $0.1 \mathrm{M}-\mathrm{CaCl}_{2}$, $37 \%$ formaldehyde and $0 \cdot 1 \mathrm{M}$-glycine ( $\mathrm{pH} 10 \cdot 4)$. Sections were then washed twice for $30 \mathrm{~s}$ each with $\mathrm{Tris}-\mathrm{Ca}^{2+}$ prerinse solution before being incubated for $90 \mathrm{~min}$ at $37^{\circ} \mathrm{C}$ in the incubation solution containing $0 \cdot 1 \mathrm{M}$-glycine buffer, $0 \cdot 18$ $\mathrm{M}-\mathrm{CaCl}_{2}$ and $0 \cdot 1 \mathrm{M}-\mathrm{ATP}(\mathrm{pH} 9 \cdot 4)$. After incubation, sections were washed four times in $0 \cdot 1 \mathrm{M}-\mathrm{CaCl}_{2}$ solution, rinsed for $3 \mathrm{~min}$ in $2 \% \mathrm{CoCl}_{2}$ solution and then rinsed four times in distilled $\mathrm{H}_{2} \mathrm{O}$. Sections were stained in $1 \%$ Azure A for $28 \mathrm{~s}$ then rinsed continuously under tap water for $5 \mathrm{~min}$. Sections were then rinsed once with distilled $\mathrm{H}_{2} \mathrm{O}$ and dehydrated through $70 \%, 95 \%$ and absolute ethanol solutions. Finally, sections were washed with Histoclear for $1 \mathrm{~min}$ then mounted with DPX Mounting medium.

\section{NADH-tetrazolium reductase staining}

The NADH-tetrazolium reductase staining method determines the oxidative capacity of the fibres (Brumback \& Leech, 1984), with oxidative fibres (types I, IIA and IIX) staining a grey/black and non-oxidative (i.e. purely glycolytic, type IIB) fibres failing to stain (i.e. are white). It should be noted that this provides measures of oxidative fibres but not 
glycolytic fibres, since some of the fibres that stain positively for oxidative metabolism (i.e. type IIA and IIX) will also have some glycolytic metabolism. Briefly, sections were left at room temperature for a standardised period of $2 \mathrm{~min}$ before they were incubated in Gomori Tris-HCl buffer ( $\mathrm{pH} 7 \cdot 4$ ), containing $0.5 \mu \mathrm{M}-\beta-\mathrm{NADH}$ (Park Scientific Ltd, Northampton, $\mathrm{UK}$ ) and $1.2 \mu \mathrm{M}$-nitroblue tetrazolium (VWR International, Lutterworth, Leics., UK) at $37^{\circ} \mathrm{C}$ for $30 \mathrm{~min}$. After incubation, sections were washed with distilled $\mathrm{H}_{2} \mathrm{O}$ for 1 min then dehydrated in 30, 60 and $90 \%$ acetone. Finally, sections were rehydrated in 60 and $30 \%$ acetone, rinsed in distilled $\mathrm{H}_{2} \mathrm{O}$ for $1 \mathrm{~min}$ and mounted in glycerine jelly.

\section{Image analysis}

Images of the ATPase- and NADH-stained sections were captured at $\times 20$ magnification using an Olympus BX50 microscope with a JVC (KY-F75U) digital camera. Three images were randomly taken from a transverse muscle section from the soleus and the medial gastrocnemius of each individual rat.

Muscle fibres were labelled as fast or slow (ATPase staining), or oxidative or glycolytic (NADH staining), by visual assessment of the stain. Every muscle fibre was counted within the three images and the data expressed as number of fibres $/ \mathrm{mm}^{2}$. The fibre diameter was measured for thirty stained and unstained fibres from each image. The cross-sectional area of the soleus muscle was measured using the image analysis software, with calibration via a graticule, to allow the calculation of the total number of fibres present in this muscle. This was not possible for the gastrocnemius muscle, due to the large, flat irregular shape of the muscle.

\section{Statistical analysis}

The numbers of offspring analysed were: control, $n 11$ (six male, five female); LPEarly, $n 10$ (six male, four female); LPMid, $n 10$ (four male, six female); LPLate, $n 8$ (four male, four female); LPAll, $n 7$ (three male, four female). All data were log transformed to adjust for non-normal error patterns observed in preliminary analyses of the original data and the residuals from the analyses of the log-transformed data were examined to confirm their normality. The data were analysed as a three-way linear mixed model using the residual maximum likelihood tool in Genstat release 8.1 (Lawes Agricultural Trust, Rothamsted Experimental Station, Herts., UK). The fixed model was defined as Diet $\times$ Sex $\times$ Fibre type and the random model as litter/animal. Error was thus determined at three levels: between dams, between offspring and between fibre types. All data are presented as means of the logged variables with the three standard errors of difference (SED) based on these analyses. Post hoc, paired comparisons (defined $a$ priori as part of the experimental design), such as between fibre type means from within the same sex and treatment group, were considered significant if the difference exceeded two standard errors of the difference. For comparisons among the five maternal diets, a treatment effect was considered significant if its mean differed from that of its control by more than $2.64 \times \mathrm{SED} ; 2.64$ being the critical value at $P=0.05$, df $=30$ for a Dunnett's test when comparing four treatments to the same control. $P<0.05$ was accepted as statistically significant and all standard errors of the difference had at least $30 \mathrm{df}$.

\section{Results}

\section{Soleus muscle}

There were no significant effects of maternal diet or sex of the offspring on the cross-sectional area of the soleus muscle at 4 weeks of age (data not shown). As expected, there were slightly more slow than fast fibres (Fig. 1(A)) and many more oxidative than glycolytic (non-oxidative) fibres (Fig. 1(B)) in the soleus muscles $(P<0.001$ for both). However, the total numbers of the different fibre types within the soleus muscle were affected by maternal dietary treatment. In the ATPase-stained sections (Fig. 1(A)), there was a Diet $\times$ Sex $\times$ Fibre type interaction $(P=0.004)$ which was mainly due to the total number of fast fibres being less than the total number of slow fibres in LPMid females; a difference that was not present in the control diet. In the NADH-stained sections (Fig. 1(B)), there was a Diet $\times$ Fibre type interaction $(P<0.001)$, due to there being fewer glycolytic (non-oxidative) fibres in LPEarly, LPMid and LPLate (but not LPAll) offspring than in the controls. There were no diet effects on the numbers of oxidative fibres and no effects of sex (Fig. 1(B)).

The densities of fibres (number of fibres $/ \mathrm{mm}^{2}$ ) were also altered by maternal dietary treatment. In the ATPase-stained sections (Fig. 2(A)), maternal LP diet increased the density of slow fibres in LPEarly males compared with controls, and for LPMid females, the density of fast fibres was less than the density of slow fibres; a difference that was not present in the control diet $(P=0.004$ for Diet $\times$ Sex $\times$ Fibre type interaction). In the NADH-stained sections (Fig. 2(B)), there was a decrease in density of glycolytic fibres in LPEarly, LPMid and LPLate (but not LPAll) compared with control offspring $(P<0.001$ for Diet $\times$ Fibre type interaction $)$, with no effect of sex.

There were few effects on fibre diameters in the soleus muscle. In the ATPase-stained sections (data not shown), the only significant differences were between fibre types (slow had larger diameters than fast, $P<0.001$ ) and the sexes (males had larger diameters than females, $P=0 \cdot 041$ ). The observation that slow fibres were larger than fast fibres would appear surprising. However, it must be remembered that these are immature neonates and not mature adults. A number of studies, especially in pigs (see Brameld et al. 1998, 2003), indicate that primary fibres (that tend to form slow fibres initially) are often larger than secondary fibres (that tend to form fast fibres initially). In the NADH-stained sections (data not shown), the complete lack of glycolytic (non-oxidative) fibres in some groups of rats meant that statistical analyses of diameters was only possible for oxidative fibres. There were no significant differences observed on the diameters of oxidative fibres (data not shown).

\section{Gastrocnemius muscle}

As expected, gastrocnemius muscles had a much greater density $(P<0.001$ for both) of fast (Fig. 3(A)) and glycolytic 

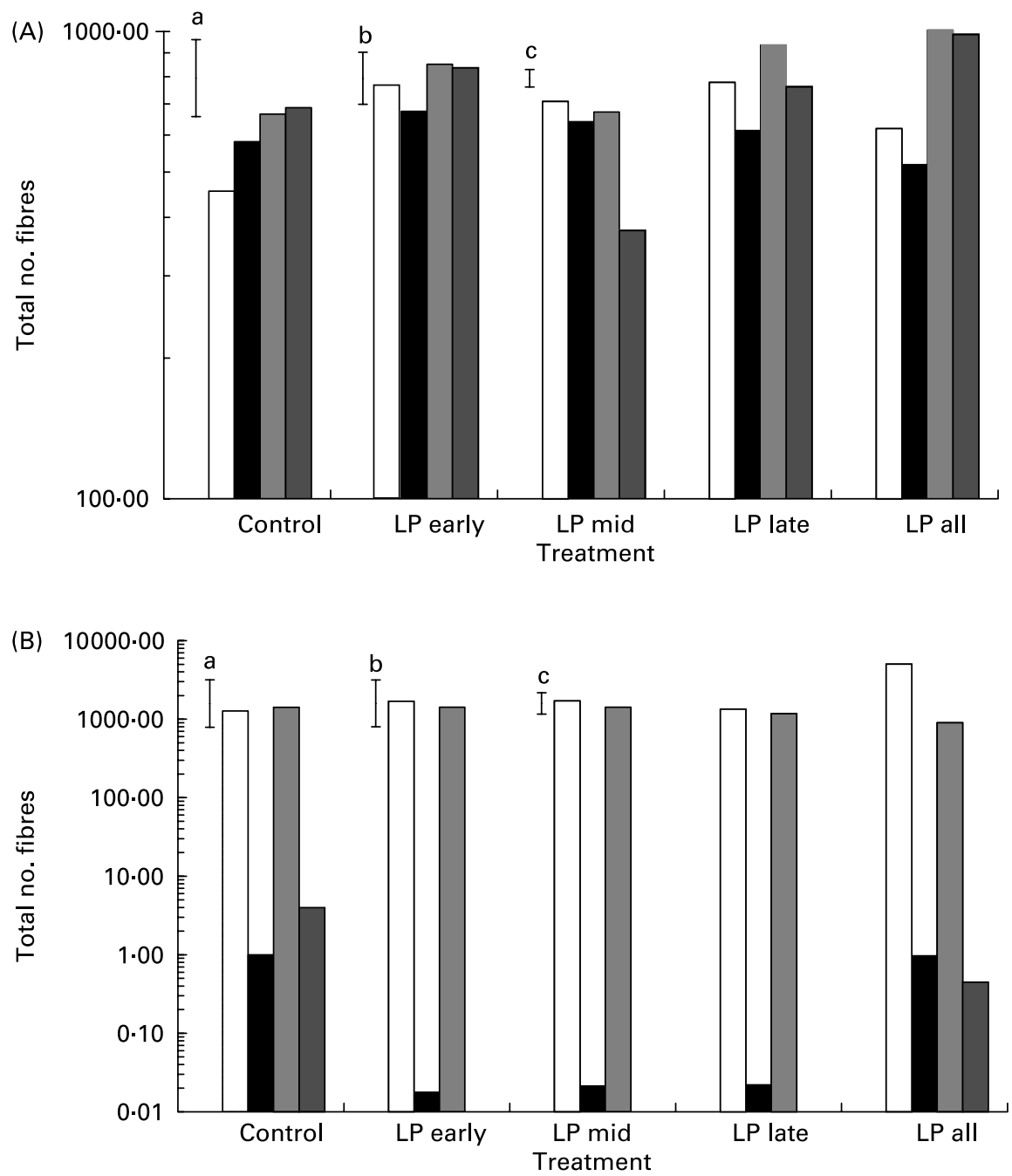

Fig. 1. Total numbers of slow ( $\square$, male; $\square$, female) and fast ( $\boldsymbol{\square}$, male; $\square$, female) fibres (A) and oxidative ( $\square$, male; $\square$, female) and glycolytic ( $\boldsymbol{\square}$, male; $\square$, female) fibres (B) in soleus from 4-week-old rats. Pregnant rats were fed a control or low-protein (LP) diet at early (days 0-7 gestation, LPEarly), mid (days 8-14, LPMid), late (days 15-22, LPLate) or throughout gestation (days $0-22$, LPAll). Values are log predicted means with the three error bars representing the standard errors of difference for comparing means on (a) different diets (between dams), (b) same diet different sex (between offspring) and (c) same diet same sex (between fibre types).

(non-oxidative) fibres (Fig. 3(B)). As observed in the soleus muscle, the densities of fibres (number of fibres $/ \mathrm{mm}^{2}$ ) within the gastrocnemius muscle were altered by maternal dietary treatment. In the ATPase-stained sections (Fig. 3(A)), there was an increase in the density of slow fibres in LPAll offspring, particularly in males $(P<0.001$ and $P=0.024$ for Diet $\times$ Fibre type and Diet $\times$ Sex $\times$ Fibre type interactions, respectively). In the NADH-stained sections (Fig. 3(B)), the density of oxidative fibres was reduced in LPMid compared with control offspring $(P=0.019$ for Diet $\times$ Fibre type interaction), with no sex effects observed.

There were very few differences in fibre diameters, with the only difference in the ATPase-stained sections (data not shown) being that slow fibres were larger than fast $(P<0 \cdot 001)$. In the NADH-stained sections (data not shown), glycolytic fibres were larger than oxidative $(P<0 \cdot 001)$ and there was a Diet $\times$ Sex interaction $(P=0.048)$, such that fibre diameters were reduced by maternal LP diet in LPEarly males.

\section{Discussion}

The main finding from the present study is confirmation that our working hypothesis appears to be at least partially correct, in that the maternal LP diet reduced the numbers and density of fast and glycolytic fibres in the soleus muscle mainly when fed during mid- to late gestation. Interestingly, a similar effect was also observed in the gastrocnemius muscle, except that this time the reduction was in the density of oxidative fibres. The data suggest that only secondary (fast) fibres are affected but that they mainly form glycolytic (presumably type IIB) fibres in the soleus muscle, whereas they normally form oxidative (presumably type IIA or IIX) fibres in the gastrocnemius muscle. The observation that the fibres affected are a minority population in the two muscles may indicate that the muscle undergoes adaptations such that the reductions occur in the least essential fibre types. Surprisingly, when protein restriction was applied throughout gestation, almost all muscle phenotypic parameters measured were comparable to 


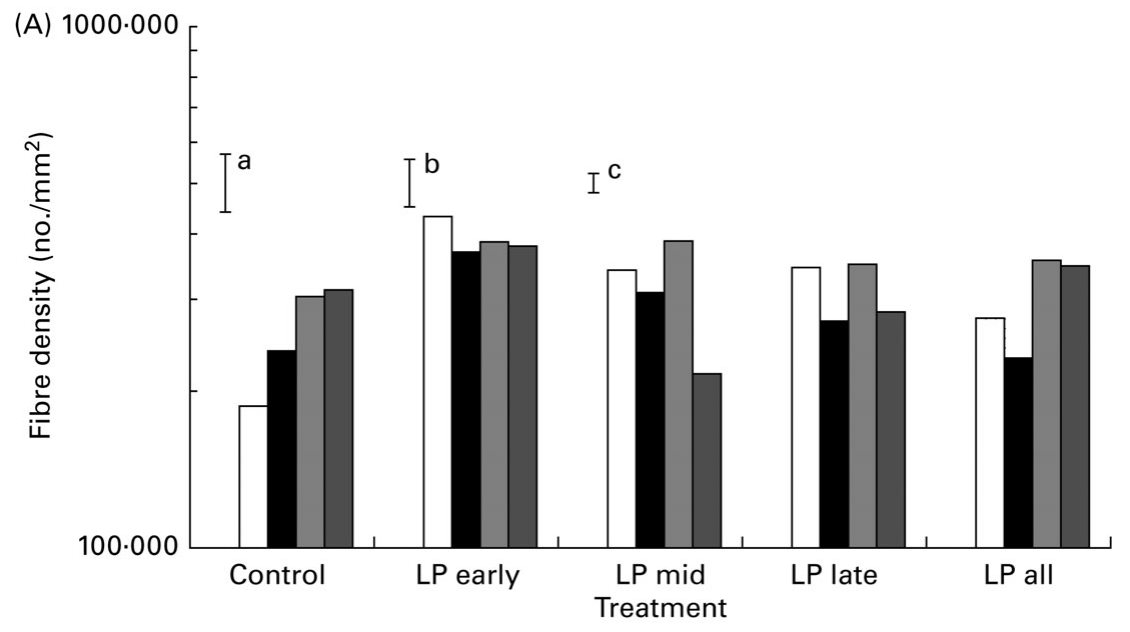

(B)

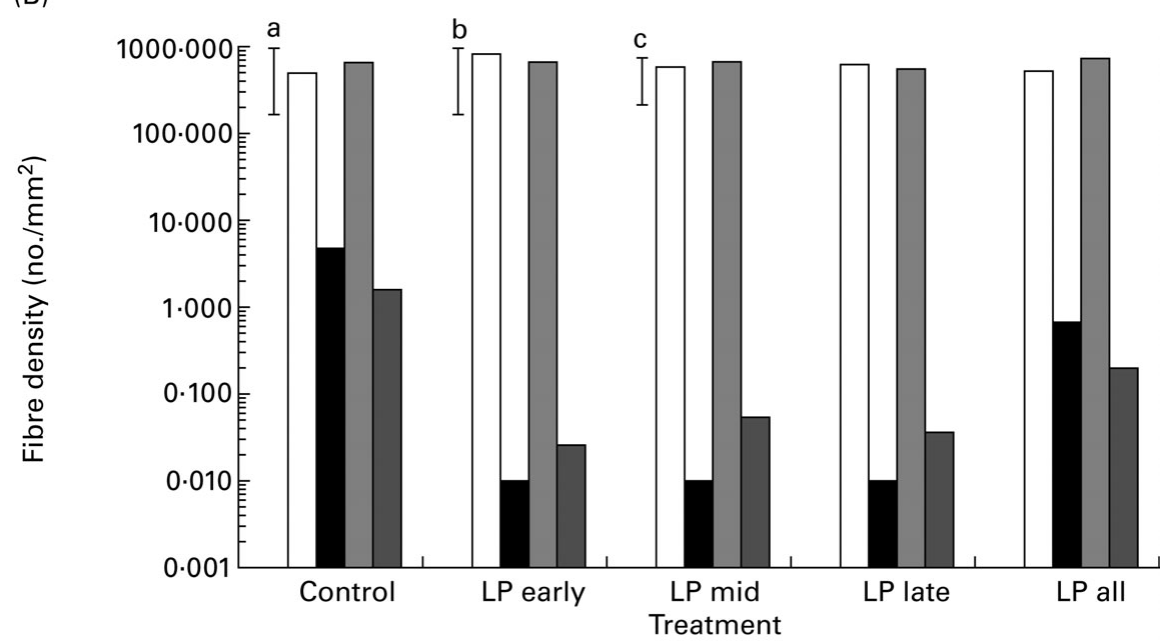

Fig. 2. Densities (number of fibres $/ \mathrm{mm}^{2}$ ) of slow ( $\square$, male; $\square$, female) and fast ( $\square$, male; $\square$, female) fibres (A) and oxidative ( $\square$, male; $\square$, female) and glycolytic ( $\mathbf{\square}$, male; $\boldsymbol{\square}$, female) fibres (B) in soleus from 4-week-old rats. Pregnant rats were fed a control or low-protein (LP) diet at early (days 0-7 gestation, LPEarly), mid (days 8-14, LPMid), late (days 15-22, LPLate) or throughout gestation (days 0-22, LPAll). Values are log predicted means with the three error bars representing the standard errors of difference for comparing means on (a) different diets (between dams), (b) same diet different sex (between offspring) and (c) same diet same sex (between fibre types).

controls. It thus appears that a mild but sustained protein restriction during gestation has very little effect on the fetal programming of muscle phenotype and the animals are able to compensate to minimise or abolish any effect on the offspring. However, changing levels of maternal protein intake during gestation appear more detrimental for the offspring, suggesting that muscle development is not sensitive to mild maternal protein restriction per se, but is sensitive to varying levels of maternal protein intake, particularly when these variations occur during mid- and late gestation. The present finding is novel and requires further investigation.

Previous work in rats has shown inconsistent effects of prenatal undernutrition on skeletal muscle characteristics. However, the studies vary in terms of the level of undernutrition used, the period of gestation targeted, and the particular muscle(s) studied. For example, studies include reductions to $50 \%$ (Bayol et al. 2004), $40 \%$ (Bayol et al. 2004) or even $30 \%$ (Wilson et al. 1988) of dietary energy requirements. Bayol et al. (2004) found no difference in the total number of fibres in semitendinosus muscle of rats at weaning when the maternal diet was restricted to 50 or $40 \%$ of the control diet throughout pregnancy. However, they did see reductions in the number of nuclei, particularly at the highest $(40 \%)$ restriction (Bayol et al. 2004). In contrast, Wilson et al. (1988) found a 19-34\% decrease of total fibre numbers in soleus and lumbrical muscles in newborn rats undernourished to $30 \%$ of the control diet throughout pregnancy, as a result of reduced secondary fibre formation. This demonstrates the importance of the level of undernutrition in whether or not changes are observed and also that there may be differences between specific muscles. Interestingly, the numbers of fibres present in the lumbrical muscle (Wilson et al. 1988) were increased by nutritional rehabilitation of the lactating mother between 7 and $28 \mathrm{~d}$ post-partum, whereas the numbers of fibres in the soleus muscle were unaffected by the nutritional rehabilitation, again suggesting differences between specific muscles in terms of critical periods of responsiveness.

Further studies in other species have tried to identify critical periods of pregnancy when muscles may be susceptible to dietary intervention. For example, studies suggest that early 

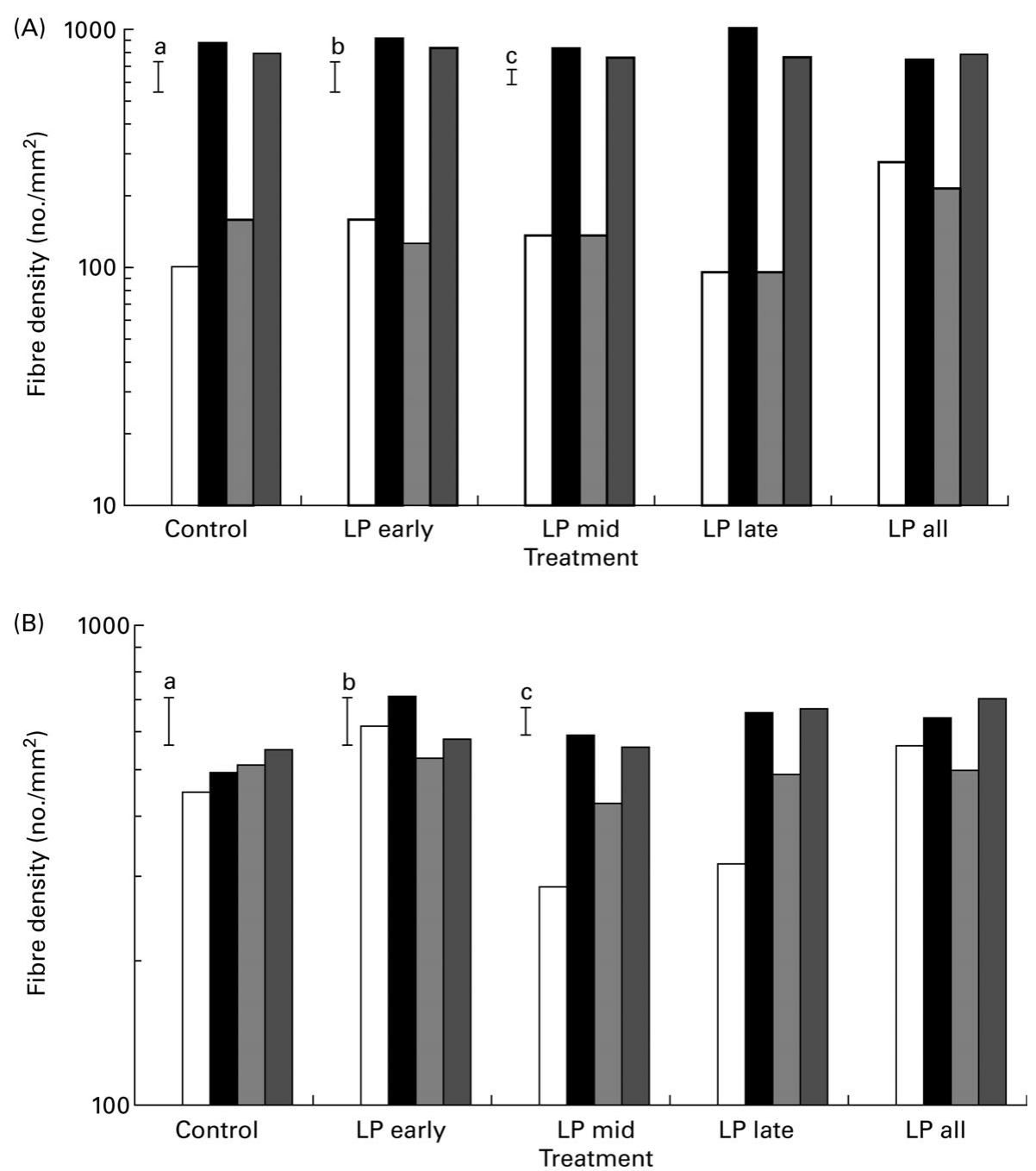

Fig. 3. Densities (number of fibres $/ \mathrm{mm}^{2}$ ) of slow ( $\square$, male; $\square$, female) and fast ( $\square$, male; $\square$, female) fibres (A) and oxidative ( $\square$, male; $\square$, female) and glycolytic ( $\square$, male; $\square$, female) fibres (B) in gastrocnemius from 4-week-old rats. Pregnant rats were fed a control or low-protein (LP) diet at early (days $0-7$ gestation, LPEarly), mid (days 8-14, LPMid), late (days 15-22, LPLate) or throughout gestation (days 0-22, LPAll). Values are log predicted means with the three error bars representing the standard errors of difference for comparing means on (a) different diets (between dams), (b) same diet different sex (between offspring) and (c) same diet same sex (between fibre types).

to mid-pregnancy may be the critical time for muscle fibre formation in sheep (Nordby et al. 1987; Fahey et al. 2005). In guinea pigs (Dwyer et al. 1995), maternal undernutrition (60\% of controls) between days 2 and 25 of gestation, days 25 and 68 (term) or throughout gestation (but not between days 2 and 15) all resulted in reduced total number of fibres in the biceps brachii muscle, but there were no effects on soleus muscle. Again this demonstrates that different muscles may respond differently to prenatal insults. Further studies in guinea pigs (Dwyer \& Stickland, 1994) indicated that supplementation of the restricted diet to replenish the protein or carbohydrate contents overcame the effects of the dietary restriction, with no effect of fat supplementation. Although there are very few similar studies investigating what components of the diet are important, the work in guinea pigs suggests dietary protein and/or carbohydrate are particularly important.

The present study therefore aimed to (1) investigate the effects of manipulating the dietary protein content of the diet and (2) test the hypothesis that the critical stage of gestation at which prenatal undernutrition influences the fibre type composition of rat muscles is during mid- to late pregnancy. The feeding of a LP diet in rat pregnancy is a wellestablished protocol for the study of prenatal programming effects upon lifespan, cardiovascular function, feeding behaviour and glucose homeostasis (Langley-Evans, 2006). In the present study the maternal LP diet was shown to influence the total numbers of fibres (soleus only) and density of fibres in the soleus and gastrocnemius muscles from rats at 4 weeks of age. Low prenatal protein particularly in mid(but not throughout) pregnancy was found to reduce the total numbers and densities of fast/glycolytic fibres formed in soleus, whereas in gastrocnemius muscle, the density of oxidative fibres was reduced by maternal LP during mid-gestation. This would suggest that dietary protein is important, since the LP was achieved by increasing the carbohydrate content of the diet in order for it to be isoenergetic with the control diet. 
In the present experiment, the two muscles studied appeared to have similar critical periods of susceptibility to dietary manipulation, although it might be suggested that soleus develops slightly earlier than gastrocnemius. Previous studies in rats (Wilson et al. 1988) and sheep (Greenwood et al. 2000; Fahey et al. 2005) indicate that prenatal undernutrition does not affect all muscles equally, or at the same stage of development. Hence, the phases of proliferation, differentiation and fibre formation of different muscles probably occur at slightly different times during gestation, and may even include the neonatal period before weaning for rats (Wilson et al. 1988). The timing of muscle fibre formation and therefore the critical periods for susceptibility to dietary manipulations also appear to differ between mammalian species (Brameld et al. 2003), although they all appear to relate to the proliferation period immediately before differentiation leading to major fibre formation.

Whether these effects are permanent or not remains to be established. To date there appear to be no studies that have investigated the long-term effects of maternal undernutrition, with the age studied being limited to fetal, neonatal or relatively young offspring. More studies in older offspring are therefore required. In addition, what the physiological impact of a reduced number of fibres in one or more skeletal muscles might be upon the animal is not clear. A similar maternal LP rat model resulted in insulin resistance in adult male offspring (Ozanne et al. 2003) and was associated with reduced levels of protein kinase $\mathrm{C}$ zeta in skeletal muscle, although the specific muscle studied is unclear. Whether these changes are due to alterations in muscle fibre composition requires further study. It has been known for a number of years that skeletal muscle accounts for a large proportion (approximately 20-30\%) of whole-body energy expenditure or BMR (Helge et al. 1999). In rats, changes in muscle fibre characteristics are associated with susceptibility (Mrad et al. 1992) or resistance (Mrad et al. 1992; Suwa et al. 2002) to obesity. We (JE Mallinson \& JM Brameld, unpublished results) have recently shown that chronic $(7 \mathrm{~d})$ treatment of rats with thyroid hormone $\left(\mathrm{T}_{3}\right)$ significantly increased resting energy expenditure (measured as whole-body oxygen consumption) as well as increasing the proportion of fast (type IIA or IIB) fibres in both soleus and tibialis anterior muscles. $\mathrm{T}_{3}$ is well recognised as increasing BMR and has previously been shown to increase the proportion of type IIA and IIB fibres in rat soleus muscle (Caiozzo et al. 1998). Based on this information, we would predict that energy expenditure by the soleus muscle would be reduced, due to the reduction in the numbers of fast/glycolytic fibres. However, the opposite or no effect might be predicted for the gastrocnemius muscle, where the reduction was in oxidative fibres. It would therefore be of interest to determine the effect of low maternal protein exposure during mid- to late pregnancy on the BMR of the offspring, as well as muscle fibre characteristics. We still do not understand what characteristics of skeletal muscle are responsible for its relationship with BMR. Severe undernutrition (to $30 \%$ of requirements) throughout pregnancy, similar to that used by Wilson et al. (1988) to demonstrate effects on muscle fibre numbers, has been shown to programme significant obesity in rodents without any need for postnatal dietary challenge (Vickers et al. 2000).
The present study agrees with our working hypothesis in that maternal LP exposure during mid- to late pregnancy reduced the numbers of fibres formed within soleus and gastrocnemius muscles of young rats, albeit with differences in terms of the fibre types affected. It appears that these effects are on secondary fibres, but that these give rise to slightly different fibre types in different muscles. Whether these changes persist into adulthood or impact upon insulin sensitivity, whole-body energy expenditure or body composition remains to be established.

\section{Acknowledgements}

The animal trial reported in this study was supported by a British Heart Foundation project grant. J. Mallinson was sponsored by the University of Nottingham.

\section{References}

Bayol S, Jones D, Goldspink G \& Stickland NC (2004) The influence of undernutrition during gestation on skeletal muscle cellularity and on the expression of genes that control muscle growth. $\mathrm{Br} J$ Nutr 91, 331-339.

Bellinger L \& Langley-Evans SC (2003) Low protein feeding in the rat impairs maternal adaptation to early pregnancy. Pediatr Res 53, P501.

Bellinger L, Sculley DV \& Langley-Evans SC (2006) Exposure to undernutrition in fetal life determines fat distribution, locomotor activity and food intake in ageing rats. Int $J$ Obes 30, 729-738.

Brameld JM, Buttery PJ, Dawson JM \& Harper JMM (1998) Nutritional and hormonal control of skeletal-muscle cell growth and differentiation. Proc Nutr Soc 57, 207-217.

Brameld JM, Fahey AJ, Langley-Evans SC \& Buttery PJ (2003) Nutritional and hormonal control of muscle growth and fat deposition. Arch Anim Breeding (Arch Tierz) 46, 143-156.

Brumback RA \& Leech RW (1984) Color Atlas of Muscle Histochemistry. Littleton, MA: PSG Publishing.

Caiozzo VJ, Baker MJ \& Baldwin KM (1998) Novel transitions in MHC isoforms: separate and combined effects of thyroid hormone and mechanical unloading. $J$ Appl Physiol 85, 2237-2248.

Colomb V, Petit J, Matheix-Fortunet H, Hecketsweiler B, Kaeffer N, Lerebours E, Colin R \& Lemeland JF (1995) Influence of antibiotics and food intake on liver glutathione and cytochrome P-450 in septic rats. Br J Nutr 73, 99-110.

Dwyer CM, Madgwick AJA, Ward SS \& Stickland NC (1995) Effect of maternal undernutrition in early gestation on the development of fetal myofibres in the guinea-pig. Reprod Fertil Dev 7, $1285-1292$.

Dwyer CM \& Stickland NC (1994) Supplementation of a restricted maternal diet with protein or carbohydrate alone prevents a reduction in fetal muscle fibre number in the guinea-pig. $\mathrm{Br} \mathrm{J}$ Nutr 72, 173-180.

Dwyer CM, Stickland NC \& Fletcher JM (1994) The influence of maternal nutrition on muscle fibre number development in the porcine fetus and on subsequent postnatal growth. J Anim Sci 72, 911-917.

Fahey AJ, Brameld JM, Parr T \& Buttery PJ (2005) The effect of maternal undernutrition before muscle differentiation on the muscle fiber development of the newborn lamb. J Anim Sci 83, 2564-2571.

Greenwood PL, Hunt AS, Hermanson JW \& Bell AW (2000) Prenatal and postnatal nutritional effects on neonatal sheep: II. Skeletal muscle growth and development. J Anim Sci 78, 50-61. 
Helge JW, Fraser AM, Kriketos AD, Jenkins AB, Calvert GD, Ayre KJ \& Storlien LH (1999) Interrelationships between muscle fibre type, substrate oxidation and body fat. Int $J$ Obes 23, 986-991.

Kwong WY, Wild AE, Roberts P, Willis AC \& Fleming TP (2000) Maternal undernutrition during the pre-implantation period of rat development causes blastocyst abnormalities and programming of postnatal hypertension. Development 127, 4195-4202.

Langley-Evans SC (2006) Developmental programming of health and disease. Proc Nutr Soc 65, 97-105.

Langley-Evans SC, Gardner DS \& Jackson AA (1996) Association of disproportionate growth of fetal rats in late gestation with raised systolic blood pressure in later life. J Reprod Fert 106, 307-312.

Langley-Evans SC, Lilley C \& McMullen S (2006) Maternal protein restriction and fetal growth: lack of evidence of a role for homocysteine in fetal programming. Br J Nutr 96, 578-586.

Langley-Evans SC \& Sculley DV (2006) The association between birthweight and longevity in the rat is complex and modulated by maternal protein intake during fetal life. FEBS Letts 580, 4150-4153.

Maltin CA, Delday MI, Sinclair KD, Steven J \& Sneddon AA (2001) Impact of manipulations of myogenesis in utero on the performance of adult skeletal muscle. Reproduction 122, 359-374.
Mrad JA, Yakubu F, Ding L, Peters JC, Atkinson JB \& Hill JO (1992) Skeletal muscle composition in dietary obesity-susceptible and dietary obesity-resistant rats. Am J Physiol 262, R684-R688.

Nordby DJ, Field RA, Riley ML \& Kercher CJ (1987) Effects of maternal undernutrition during early pregnancy on growth muscle cellularity, fibre type and carcass composition in lambs. $J$ Anim Sci 64, 1419-1427.

Ozanne SE, Olsen GS, Hansen LL, Tingey KJ, Nave BT, Wang CL, Hartil K, Petry CJ, Buckley AJ \& Mosthaf-Seedorf L (2003) Early growth restriction leads to down regulation of protein kinase $\mathrm{C}$ zeta and insulin resistance in skeletal muscle. J Endocrinol 177, 235-241.

Suwa M, Kumagai S, Higaki Y, Nakamura T \& Katsuta S (2002) Dietary obesity resistance and muscle oxidative enzyme activities of the fast-twitch fibre dominant rat. Int J Obes 26, 830-837.

Vickers MH, Breier BH, Cutfield WS, Hofman PL \& Gluckman PD (2000) Fetal origins of hyperphagia, obesity, and hypertension and postnatal amplification by hypercaloric nutrition. Am J Physiol Endocrinol Metab 279, E83-E87.

Wegner J, Albrecht E, Fiedler I, Teuscher F, Papstein HJ \& Ender K (2000) Growth and breed related changes of muscle fibre characteristics in cattle. J Anim Sci 78, 1485-1496.

Wilson SJ, Ross JJ \& Harris AJ (1988) A critical period for formation of secondary myotubes defined by prenatal under nourishment in rats. Development 102, 815-821. 